\title{
Linking ImageNet WordNet Synsets with Wikidata
}

\author{
Finn Årup Nielsen \\ Technical University of Denmark \\ DTU Compute \\ Kongens Lyngby, Denmark \\ faan@dtu.dk
}

\begin{abstract}
The linkage of ImageNet WordNet synsets to Wikidata items will leverage deep learning algorithm with access to a rich multilingual knowledge graph. Here I will describe our ongoing efforts in linking the two resources and issues faced in matching the Wikidata and WordNet knowledge graphs. I show an example on how the linkage can be used in a deep learning setting with real-time image classification and labeling in a non-English language and discuss what opportunities lies ahead.
\end{abstract}

\section{KEYWORDS}

Wikidata; ImageNet; WordNet; ontology alignment; machine learning

\section{ACM Reference Format:}

Finn Årup Nielsen. 2018. Linking ImageNet WordNet Synsets with Wikidata. In WWW'18 Companion: The 2018 Web Conference Companion, April 23-27, 2018, Lyon, France. ACM, New York, NY, USA, 6 pages. https://doi.org/10.1145/3184558.3191645

\section{INTRODUCTION}

Machine learning with deep neural networks has in recent years yielded interesting advances with a range of tasks. Particularly image classification models have seen advances with the first system claiming "superhuman visual pattern recognition" in a restricted and controlled setting back in 2011 [10] More recent trends have explored how knowledge bases can be utilized together with machine learning. For instance, a combined word embedding of the ConceptNet knowledge graph and the two popular word embeddings, word2vec and GloVe, could produce state-of-the-art results on word similarity tests [11], while the Wembedder system uses an embedding of the Wikidata knowledge graph in a web application with recommender system features [8].

To take advantage of knowledge graphs in machine learning we should deep-link systems, and preferably not just on the word-level but on a semantic level where the different senses of polysemic words are distinguished. One opportunity is a linkage between ImageNet through Wordnet to Wikidata.

I would like to thank Laura Rieger and Lars Kai Hansen for discussions. This research was supported by Innovation Fund Denmark through the DABAI project.

This paper is published under the Creative Commons Attribution 4.0 International (CC BY 4.0) license. Authors reserve their rights to disseminate the work on their personal and corporate Web sites with the appropriate attribution. WWW'18 Companion, April 23-27, 2018, Lyon, France

() 2018 IW3C2 (International World Wide Web Conference Committee), published under Creative Commons CC BY 4.0 License.

ACM ISBN 978-1-4503-5640-4/18/04.

https://doi.org/10.1145/3184558.3191645
Below I will describe these three resources and follow with a detailed account of issues around on-going work on matching the items across these resources. I will show statistics and describe a small machine learning application that uses the linkage between ImageNet and Wikidata, and lastly discuss issues concerning the matching and the opportunities for extending Wikidata.

\section{WORDNET, IMAGENET AND WIKIDATA}

\subsection{WordNet}

WordNet is a machine readable lexical resource that describe words. Words that are synonyms are grouped into items called synsets [6]. Each WordNet synset is associated with an Semantic Web Linked Open Data (LOD) URI. URIs for WordNet for version 3.0 has been prefixed with http://wordnet-rdf. princeton.edu/wn30/ while URIs for version 3.1 synsets are prefixed with http://wordnet-rdf.princeton.edu/wn31/. The canonical prefix has been change recently, so the part wn30 has been changed to pwn $30 .^{1}$

\section{$2.2 \quad$ ImageNet}

ImageNet [2] is a large collection of images, labeled against WordNet 3.0 and described at http://image-net.org/. According to January statistics, ImageNet contains 14,197,122 images and 21,841 indexed synsets. There are various labeling schemes. The object attributes scheme labels 400 synsets across 25 attributes. The attributes are with respect to color, pattern, shape and texture. ${ }^{2}$ ImageNet Large Scale Visual Recognition Challenge ILSVRC [9] is an image recognition challenge that started in 2010 and has been repeated for several years. It has had several tasks: image classification, single-object locatization or object detection. For the image classification task a subset with 1,000 object categories from ImageNet was selected corresponding to 1,000 WordNet synsets. ILSVRC synsets have changed through the years so only 639 have been used in all the years.

Inspired by the demonstration of the so-called AlexNet [5] where state-of-the-art image classification results were reported on the ILSVRC dataset, many publications describe the usage of the ILSVRC data for training deep learning image classification models. Researchers distribute pre-trained deep learning models based on the ILSVRC data. One such model is Resnet-50 described by [3]. High-level deep learning Python frameworks, Keras and Caffe, are able to read this

\footnotetext{
${ }^{1}$ See the discussion on the WN-USER mailing list at https://ists.princeton. edu/cgi-bin $/$ wa $? A 2=$ ind $1801 \& \mathrm{~L}=$ wn- users $\& \mathrm{~F}=\& \mathrm{~S}=\& \mathrm{P}=1719$. The old URIs are redirected.

${ }^{2}$ http://image-net.org/download-attributes
} 
model in a few lines of code. For instance, with Keras the pre-

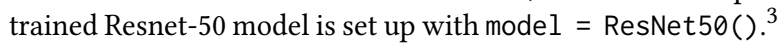
The Keras implementation will also convert the output of the trained model from its index-based value to the WordNet synset identifier.

In ImageNet the synset identifier is represented with the word class as a prefix, e.g., the synset identifier for banana is n07753592. So a (trivial) conversion is necessary to match the LOD URIs which in the banana case would be http://wordnet-rdf.princeton.edu/wn30/07753592-n, while a preview of corresponding ImageNet images is available as http://image-net.org/explore.php?wnid=n07753592.

\subsection{Wikidata}

The collaborative knowledge graph, Wikidata, at https://www. wikidata.org [12] has over 40 million items (with the identifier prefixed with "Q"), that each may be described by several thousand properties (prefixed with "P"). Wikidata properties with the data type "External identifier" is often suggested as a means to deep linking external databases. Such a method could also be applied for WordNet synset identifiers. This requires a property suggestion and some deliberation among the Wikidata editor before the external identifier property is made available for use. ${ }^{4}$ There is another way to link Wikidata with WordNet. Wikidata has a property to link to LOD URIs: the "exact match" property (P2888), corresponding to skos:exactMatch. This method can be used to link a Wikidata item to its associated WordNet synset, e.g., mashed potato (Q322787) is linked via the $\mathrm{P} 2888$ property to http://wordnet-rdf.princeton.edu/wn30/07711569-n.

Wikidata links to another lexical resource: The multilingual BabelNet ${ }^{5}$ is linked via its own external identifier property, the P2581 ${ }^{6}$ which currently is used 59.105 times. ${ }^{7}$ While WordNet is one of the resources that uses BabelNet [7], the identifier is different. For instance, "quill pen" is 00065709n in BabelNet and 04033901-n in WordNet 3.0.

Once WordNet 3.0 URIs have been entered into Wikidata, we can obtain the inverse mapping from the WordNet URI to the Wikidata item with the Wikidata Query Service (WDQS) SPARQL engine, e.g., the SPARQL query "SELECT * WHERE \{ ?item wdt:P2888

<http://wordnet-rdf.princeton.edu/wn30/04033901-n> \}" will identify Q4063215 as the Wikidata item corresponding to the WordNet concept "quill".

One advantage with Wikidata is its multilingual nature: Once we have linked a WordNet synset to a Wikidata item we can record labels in multiple languages, and in many cases the labels are already present. For instance, "quill pen" is currently labeled for 33 languages. The corresponding number

\footnotetext{
${ }^{3}$ https://keras.io/applications/\#resnet50

${ }^{4}$ I suggested such a property, see https://www.wikidata.org/wiki/Wikidata: Property_proposal/Wordnet_synset_ID.

${ }^{5} \mathrm{http}: / /$ babelnet.org/.

${ }^{6}$ https://www.wikidata.org/wiki/Property:P2581.

${ }^{7}$ Wikidata Query Service can be queried with "SELECT (COUNT (*) AS ?count) WHERE \{ [] wdt:P2581 [] \}", see http://tinyurl.com/y8pk2cqa
}

for BabelNet is 12. It is important to note though that the multilinguality in Wikidata is certainly not complete and biased towards the major European languages [4].

Wikidata and WordNet have been defined mostly independent of each other. There are other linked semantic resources where this is also the case. Examples are some of the wordnets inspired by the original English (Princeton) WordNet [1].

\section{MATCHING WIKIDATA WITH IMAGENET}

For matching Wikidata with ImageNet, I focused on the ILSVRC-part of ImageNet synsets linking them up with the P2888 property and the http://wordnet-rdf.princeton.edu/wn30/ prefix by examining Wikidata definitions, WordNet synsets descriptions, ImageNet images linked to the WordNet synsets and sometimes the description in Wikipedia articles associated with Wikidata items. Matching Wikidata with ImageNet is for some concepts straightforward. For instance, many taxons can readily be matched. At other times the matching requires some thought. Table 1 shows examples where a match is particular difficult. Below I attempt a classification of various matching problems faced during manual linking of Wikidata items to WordNet synsets.

An ILSVRC synset is missing from Wikidata. In most of these cases, it is easy to create new Wikidata items and link them to WordNet. Examples where I could not find matching items and created new items are soup bowl and bath towel.

The matching Wikidata item is a Wikipedia disambiguation page: An example is car mirror where the English Wikipedia page "Car mirror" currently is a disambiguation page linking to two real articles "Rear-view mirror" and "Wing mirror". A solution to such a problem would be to either create a new Wikidata item for car mirror or change the definition of "Car mirror" in both Wikidata and the English Wikipedia. "Armchair is a disambiguation page in the English Wikipedia, but another item link armchair in Wikidata (Q25503439). Mixing bowl is another example where the Wikidata item is describing a disambiguation page.

There is a discrepancy between ImageNet and WordNet: For instance, sunglass (http://wordnet-rdf.princeton.edu/ wn30/04355933-n) is described as a "a convex lens that focuses the rays of the sun; used to start a fire". However, the associated ImageNet images are better described with the http://wordnet-rdf.princeton.edu/wn31/104296228-n synset (dark glasses, shades, sunglasses) described with "spectacles that are darkened or polarized to protect the eyes from the glare of the sun". This issue was previously noted by Tom White on Twitter in September 2017..$^{8}$ In the same context he also reported differences between the sunglass and the sunglasses images. A similar confusion occurs for maillot, where Wordnet has two synsets: "a woman's one-piece bathing suit" and "tights for dancers or gymnasts". For the latter synset, ImageNet displays images for one-piece bathing suits. Another example is monitor described as "Electronic equipment

\footnotetext{
${ }^{8}$ Tom White (@dribnet). aha: "sunglass" is "burning glass", ...https://twitter. com/dribnet/status/904133638257655808 (3 September 2017).
} 


ImageNet
n07930864: Mostly (coffee) cups, i.e., “a
small open container usually used for
drinking; usually has a handle”, but also
a few champagne glasses.
n07565083: Printed cards with informa-
tion about dishes served.
n03742115: Image of bathroom cabinets.

n03832673: Images linked directly to n03832673 show computer notebooks, but the planner child synset shows paper notebooks.

n04152593: Cathode-ray tube (CRT) screens as well as some flatscreens. Often the full apparatus, - not just the display. Mostly computer screens.

n04355933: Mostly (all?) protective eyewear.

n03944341: Toy pinwheels
WordNet
Wikidata

Cup; "A punch served in a pitcher instead of a punch bowl", - the 6th sense of cup.

Menu: "The dishes making up a meal".

Medicine chest, medicine cabinet; "Cabinet that holds medicines and toiletries"

Notebook is a notebook computer, but the WordNet hierarchy displayed on the ImageNet homepage shows planner n03956785 as a hyponym and this synset is described as "A notebook for recording appointments and things to be done, etc." There is a separate item for laptop.

Screen, CRT screen: "The display that is electronically created on the surface of the large end of a cathode-ray tube".

Sunglass; "A convex lens that focuses the rays of the sun; used to start a fire"

Pinwheel; "A wheel that has numerous pins that are set at right angles to its rim". There is another WordNet synset for the toy: n03944138 also called "pinwheel wind collector".
? Q1121224 is an item for the parent synset (punch). There seems to be none for the punch cup synset.

? ImageNet-consistency could link to Q658274. There does not seem to be an item corresponding to the WordNet sense.

Rather than medicine chest, the WordNet synset corresponds to bathroom cabinet (Q4869069)

? The closest Wikidata item may be Q3962 with laptop as the current English label. There does not seem to be an individual item for computer notebook.

? Q5290 is computer monitor or screen, and not necessarily CRT-based. Q1736293 is cathode ray tube screen fitting WordNet but less so ImageNet.

? Q368027 is "burning glass" corresponding to the WordNet sense, but quite different from the examples in ImageNet.

? Pinwheel is a disambiguation page in English Wikipedia, while "Pinwheel (toy)" corresponds to ImageNet usage and n03944138.

Table 1: Matching between ImageNet, WordNet and Wikidata. Some examples of the difficulties with aligning the resources.

that is used to check the quality or content of electronic transmissions". At ImageNet's n03782006, the images shown are broader with many displaying computer monitors, - in Wikidata it would correspond to approximately the more general concept Q6021804 ("electronic visual display"). Related to the monitor issue is screen with the alias CRT screen and description "The display that is electronically created on the surface of the large end of a cathode-ray tube". The associated ImageNet images show many screens that are not CRT-based, but flat panel displays. One interesting discrepancy is for spider web: WordNet has two synsets for that word: "a web spun by spiders to trap insect prey" and "a web resembling the webs spun by spiders" where the latter is regarded as an artifact and the former is not found in ImageNet. ImageNet images labeled with the latter sense (n04275548) show real natural spider webs. It is obvious that webs spun by spiders resemble webs spun by spiders, but in this case the webs spun by spiders should probably not be assigned to the WordNet synset. Table 1 shows other examples of this problem, e.g., for cup (coffee cup or punch), menu (printed list or a set of servings), notebook (computer or paper book for notes) and pinwheel. Not shown in the table is picture rail ("rail fixed to a wall for hanging pictures") and its parent rail ("a horizontal bar (usually of wood or metal)"). For both these synsets ImageNet associates images of railways and trains. These cases may involve concepts with a large semantic difference. There are likely more discrepancies.

There are some difference between the WordNet and the Wikidata concepts with similar names. For instance, WordNet defines street sign as "a sign visible from the street" while Wikidata describes the same English word as a "type of traffic sign used to identify named roads" (Q1969455). The Wikidata item for traffic sign (Q170285) is described as a "symbol for people out in traffic" corresponding better to the street sign of WordNet. Another example is "tea chest" that in the 
English Wikipedia (the only Wikipedia describing the concept) is explained as a case "approximate size $500 \times 500 \times 750$ millimeters" with metal edges. Wikidata links this concept to the identifier 300039143 of the Art \& Architecture Thesaurus where it is explained as "Square wooden cases usually fixed with sheet lead or tin and used for exporting tea." The English Wikipedia further extends the concept with "The term is now used more widely to indicate similarly-sized cases, including cardboard cases, produced for various home and commercial uses." In ImageNet tea chest (n04397168) are cases with tea of varying sizes corresponding well to the WordNet's general description: "Chest for storing or transporting tea". Here we have an example of the English Wikipedia describing two concepts in the same article, a specific and a general, where the general topic correspond to WordNet and ImageNet concepts.

There are a number of other cases where the matching requires an effort to resolve. These may involve multiple semantically similar items in WordNet and Wikidata: For instance, there are multiple synsets for radiator. The radiator synsets in ILSVRC (n04040759) is described as "A mechanism consisting of a metal honeycomb through which hot fluids circulate; heat is transferred from the fluid through the honeycomb to the airstream that is created either by the motion of the vehicle or by a fan". Most of the ImageNet images associated with this synset fit the description of another synset described as a "heater consisting of a series of pipes for circulating steam or hot water to heat rooms or buildings". In Wikidata there are 3 or 4 items close in semantics to radiator. WordNet distinguishes between tape player, (n04392985) and tape recorder (n04393095). In Wikidata there is only one item. Wikidata has multiple items for infant bed (infant bed, bassinet), the media archive Wikimedia Commons distinguishes between baby beds, bassinets, cradles and cribs among others. ImageNet WordNet has two bassinet synsets, one cradle, one carrycot and one crib, with four of them grouped under baby bed. ImageNet WordNet has two main mask synsets and Wikidata has also two items with the main label "mask". They differ slightly in semantics and for the n03725035 synset described as "A protective covering worn over the face", ImageNet associates images that are not necessarily protective coverings. ImageNet Wordnet has cassette and cassette tape synsets that are difficult to dissociate from their description ("a container that holds a magnetic tape used for recording or playing sound or video" vs. "a cassette that contains magnetic tape"). One is grouped under container and the other under memory device. Wikidata's cassette corresponds more to the audiocassette of ImageNet WordNet.

\section{STATISTICS}

With a SPARQL query in WDQS, we can count the number of WordNet 3.0 synsets linked from Wikidata:

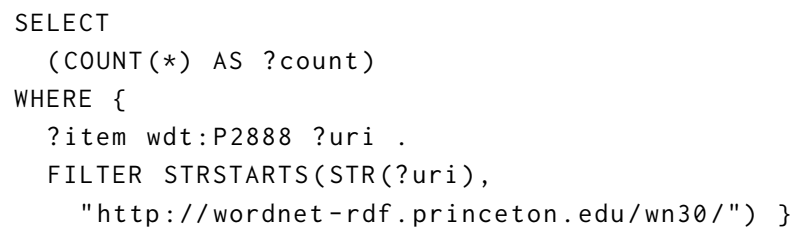

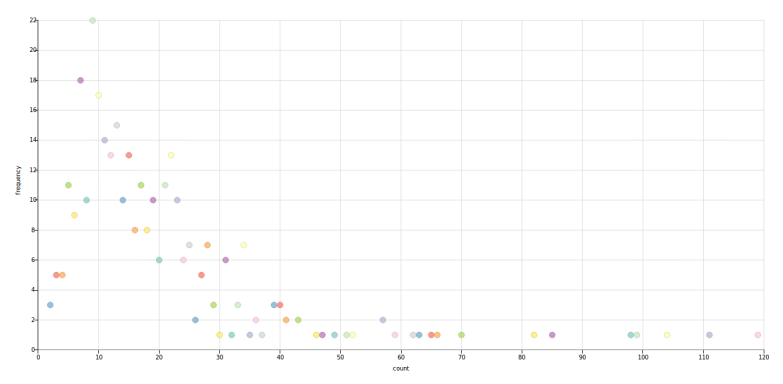

Figure 1: Plot from the Wikidata Query Service displaying the frequency of the number statements in each Wikidata item that is linked to WordNet 3.0.

The response currently yields 324 , i.e., approximately a third of the total number of ILSVRC synsets. A query for items that links to both WordNet 3.0 and BabelNet (with the P2581 property) yields 105 Wikidata items.

Wikidata items with links to WordNet synsets may have quite few properties describing the items. For instance, the only properties describing oxygen mask (Q1890958) is currently the exact match (P2888), the Wikimedia Commons category property (P373) and 3 external identifiers for Freebase, Quora and JSTOR. For loupe (Q4165197) there is only one property beyond the exact match property.

We can count the number of statements (i.e., the number of property values) for each Wikidata item with the follow WDSQ SPARQL query:

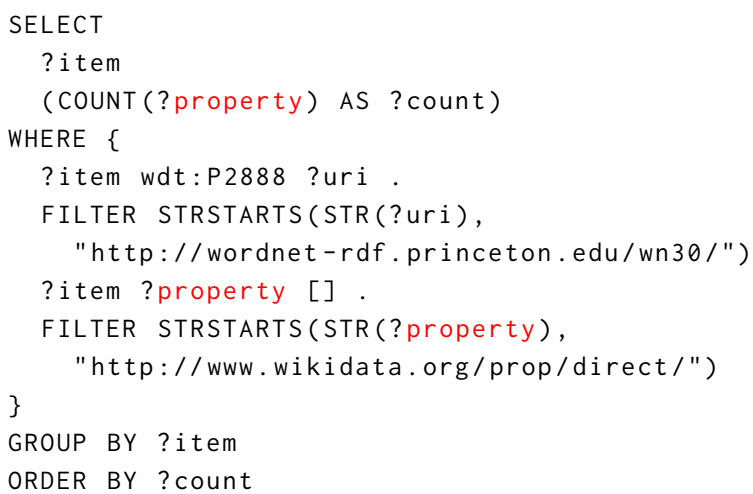

The sorted list shows that the WordNet 3.0-linked Wikidata items with the most statements are typically various taxons: Dog, bald eagle and goldfish each has over 100 statements. On the other hand, inanimate objects like bathroom cabinet, loupe, cradle and cathode ray tube screen as well as newly created pill bottle and bath towel have three or less statements, - including the statement(s) linking to WordNet.

We can get an overview of the counts by plotting the statement counts with the following SPARQL, producing a histogram-like plot:

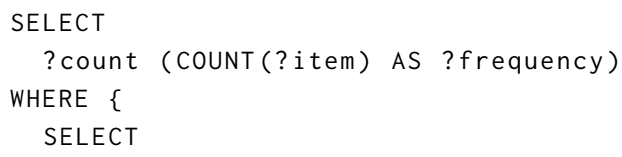




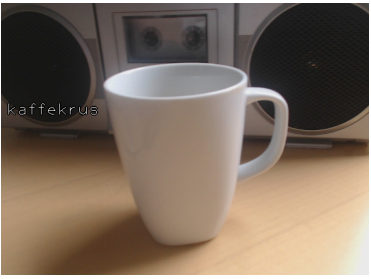

(a) "Coffee mug" (b) "Washing machine"

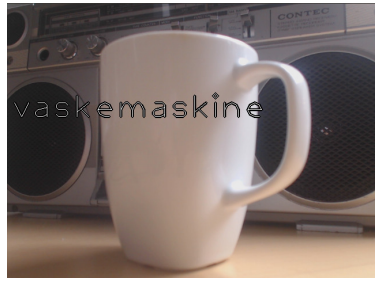

Figure 2: Two screenshots of an application using the Keras Resnet-50 model for image classification of camera images with labels translated to Danish via Wikidata. A coffee mug is in front of a tape recorder on a desk. In one case the label reads "kaffekrus" (coffee mug), in the other case-a less successful classificationit reads "vaskemaskine" (washing machine).

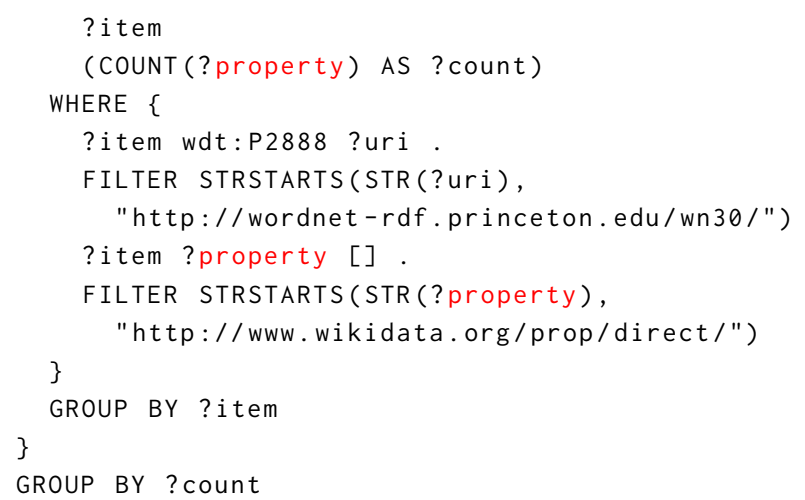

Figure 1 shows the scatter plot output from WDQS. The most frequent number of statements are 9. Examples on Wikidata items with this number of statements are filing cabinet, Miniature Pinscher (a dog bred), table lamp and cowboy hat

\section{EXAMPLE APPLICATION}

I created an example application that would use Wikidata in an image classification setting. The application was implemented in Python using the Keras deep learning framework with a TensorFlow backend. Images were captured real-time from a USB camera with OpenCV. For ImageNet-based image classification, I used the Resnet-50 pre-trained neural network in the form of the ResNet50 Keras implementation. Before the camera images were feed to the neural model, they were cropped and preprocessed with the ResNet 50 preprocessing method following the example code provided by François Chollet at https://github.com/fchollet/deep-learning-models. The index-based output of the neural network was converted to the associated WordNet synset identifier with the decoder provided by Keras. Only the index associated with the highest probability in prediction was used. This identifier was used for a least-recently-used cached lookup in WDQS to identify the corresponding Wikidata item. The Danish label from Wikidata was overlayed on the camera image and the resulting image displayed on the computer screen via an OpenCV function.
The font size was dynamically adjusted to indicate the probability of the classification. Figure 2 shows screenshots of the running application.

Running on a desktop computer with an NVIDIA Quadro K620, the update rate with camera capture, preprocessing, neural computation and display would take around 80 milliseconds for TensorFlow running on the GPU, while around 180 milliseconds if only the CPU was used. If the query returning the label was not cached, then the update would take a few hundred milliseconds extra while querying WDQS.

\section{DISCUSSION}

I have pointed to several cases of discrepancies between WordNet and associated ImageNet images. This is a problem if the hierarchy of WordNet or Wikidata is used for semantic background knowledge in an image classification setting. Given that ImageNet is used for benchmarking it may be less optimistic to think that the annotation will be changed in ImageNet. How can Wikidata record that certain WordNet synsets are not well represented in ImageNet? One way would be to make a dedicated property for ImageNet synset identifiers in Wikidata, - independently of WordNet. These would enable Wikidata to record ImageNet's discrepancies, e.g., with the "deprecated" feature or by a suitable qualifier attached to the property. A dedicated property may also make certain SPARQL query faster as it would not require the use of the FILTER SPARQL keyword to distinguish between different LOD URI prefixes.

The low number of statements for Wikidata items linked to ILSVRC classes via WordNet synsets poses a problem if we want to utilize these statements to give contexts to the categories identified with ImageNet-trained image classifiers. Even for $d o g$-the Wikidata items with the most statements among those items linked to WordNet currently-the number of relevant statements to support a semantic characterization is low: Many of the statements are for the property taxon common name (which is just a label) and the number of statements which links to other statements and characterizes the concept of $d o g$ well may be argued to be less than 10: It is a subclass of domesticated animal and pet; it can be used as a pet, for hunting and guarding and as a service animal; and it can produce a sound that is a bark. We may contrast that to ConceptNet where there are more statements (assertions), but they are also less formal: According to ConceptNet a dog has a big heart, brains, fleas, four legs, fur, nose, one mouth, paws, penis, teeth, two ears; a dog can be located in the backyard, in bed, on a couch or desk, in a doghouse, etc.; and it is capable of barking, biting and fighting a cat among lots of other activities. ${ }^{9}$ There are opportunities to extend Wikidata with statements that better characterize its items, though it is not readily clear how this can be done. For instance, how should we represent that a dog typically has fur and four legs? Or that in some contexts it forms an antonym with cat? One property that could be used more extensively is has quality (P1552) for non-material

\footnotetext{
${ }^{9}$ These statements come from conceptnet-assertions-5.5.0.csv.gz which can be downloaded from https://s3.amazonaws.com/conceptnet/precomputed-data/ 2016/assertions/conceptnet-assertions-5.5.0.csv.gz, see https://github.com/ commonsense/conceptnet5/wiki/Downloads.
} 
characteristics. Physical objects may be characterized by, e.g., height and weight, but how should we describe the size of, say, a coffee mug: By an average height and weight over a set of coffee mugs? If yes, then where would we get that data from? Wikidata items may be used to characterize other items, e.g., visual works may be described by what they depict and written works what they are about. For instance, Wikidata can describe that washing machine is the main topic of a set of scientific articles. Yet the examples we have in Wikidata now are mostly medical articles describing accidents with washine machines. It not obvious how such annotations can be used with benefit in ImageNet-based machine learning system.

Regardless of the issues around matching ImageNet synsets with Wikidata items and the questions raised here in the discussion, I see interesting opportunities in the combination of machine learning and a collaborative knowledge base such as Wikidata.

\section{REFERENCES}

[1] Bond, F., Vossen, P., McCrae, J. P., and Fellbaum, C. CIli: the Collaborative Interlingual Index. Proceedings of the Eighth Global WordNet Conference (January 2016), 50-57.

[2] Deng, J., Dong, W., Socher, R., Li, L.-J., Li, K., AND Li, F.-F. ImageNet: A large-scale hierarchical image database. 2009 IEEE Conference on Computer Vision and Pattern Recognition (June 2009).
[3] He, K., Zhang, X., Ren, S., and Sun, J. Deep Residual Learning for Image Recognition. 2016 IEEE Conference on Computer Vision and Pattern Recognition (December 2015), 770-778.

[4] Kaffee, L.-A., Piscopo, A., Vougiouklis, P., Simperl, E., CArr, L., AND Pintscher, L. A Glimpse into Babel: An Analysis of Multilinguality in Wikidata. Proceedings of the 13th International Symposium on Open Collaboration (August 2017).

[5] Krizhevsky, A., Sutskever, I., ANd Hinton, G. Imagenet classification with deep convolutional neural networks. Advances in Neural Information Processing Systems 25 (December 2012), 1097-1105.

[6] Miller, G. A. WordNet: a lexical database for English. Communications of the ACM 38 (November 1995), 39-41.

[7] Navigli, R., And Ponzetto, S. P. BabelNet: building a very large multilingual semantic network. Proceedings of the 48th Annual Meeting of the Association for Computational Linguistics (July 2010), 216-225.

[8] Nielsen, F. A. Wembedder: Wikidata entity embedding web service.

[9] Russakovsky, O., Deng, J., Su, H., Krause, J., Satheesh, S., Ma, S., Huang, Z., Karpathy, A., Khosla, A., Bernstein, M., Berg, A. C., And Li, F.-F. ImageNet Large Scale Visual Recognition Challenge.

[10] Schmidhuber, J. Deep learning in neural networks: an overview. Neural Networks 61 (October 2014), 85-117.

[11] Speer, R., Chin, J., And Havasi, C. ConceptNet 5.5: An Open Multilingual Graph of General Knowledge. Proceedings of the Thirty-First AAAI Conference on Artificial Intelligence (December 2016), 4444-4451.

[12] VRANDEČIĆ, D., AND KRÖTZSCH, M. Wikidata: a free collaborative knowledgebase. Communications of the ACM 57 (October 2014), 78-85. 